\title{
Colossal enhancement of upper critical fields in noncentrosymmetric heavy fermion superconductors near quantum criticality: $\mathrm{CeRhSi}_{3}$ and $\mathrm{CeIrSi}_{3}$
}

\author{
Y. Tada * N. Kawakami, and S. Fujimoto \\ Department of Physics, Kyoto University, Kyoto 606-8502, Japan
}

\begin{abstract}
Strong coupling effects on the upper critical fields $H_{c 2}$ along the $c$-axis in the noncentrosymmetric heavy fermion superconductors near quantum criticality $\mathrm{CeRhSi}_{3}$ and $\mathrm{CeIrSi}_{3}$ are examined. For sufficiently large spin-orbit interactions due to the lack of inversion symmetry, $H_{c 2}$ is mainly determined by the orbital depairing effects. From microscopic calculations taking into account the strong spin fluctuations, we show that $H_{c 2}$ is extremely enhanced as the system approaches the quantum critical point, resulting in $H_{c 2} \sim 30 \mathrm{~T}$ even in the case with the low transition temperature $T_{c}(H=0) \sim 1 \mathrm{~K}$, which well explains the huge $H_{c 2}$ observed in the recent experiments. Our results reveal intrinsic and universal properties common to strong coupling superconductivity caused by spin fluctuations near quantum criticality.

PACS numbers:
\end{abstract}

Quantum criticality is a phenomenon emerging in the vicinity of a second-order phase transition at sufficiently low temperatures governed not by thermal fluctuations but by quantum fluctuations. There has been accumulated evidence that quantum criticality plays an essential role in unconventional superconductivity realized in strongly correlated electron systems such as heavy fermion compounds and high- $T_{c}$ cuprates 1 . In particular, it has been proposed by several authors that magnetic critical fluctuations mediate Cooper pairing in some classes of unconventional superconductors $2,3,4,5,6,7$. In this scenario, it is expected that the pairing interaction is explosively enhanced just at the quantum critical point (QCP), though such enhancement has not been observed so far in real systems, partly because the QCP at zero temperature associated with the magnetic order is veiled by the superconducting phase. In this respect, the recent experimental studies on the upper critical fields of noncentrosymmetric heavy fermion superconductors $\mathrm{CeRhSi}_{3}{ }^{8,9}$ and $\mathrm{CeIrSi}_{3}{ }^{10,11}$ under applied pressure are very curious ${ }^{12,13}$. In these systems, the distance from the QCP is controlled by the applied pressure. The remarkable features of the experimental results are as follows: (i) As the pressure approaches a critical value, these systems exhibit extremely high upper critical fields $H_{c 2}$ which exceed the orbital limit as well as the Pauli limit estimated by the conventional BCS theory. The observed $H_{c 2} \sim 30 \mathrm{~T}$ is, surprisingly, almost comparable to those of high- $T_{c}$ systems, though the transition temperature at zero field is merely $\sim 1 \mathrm{~K}$. (ii) The increase in the upper critical field is enormously accelerated as the temperature $T$ is decreased, making a sharp contrast to any other superconductors in which the increase of $H_{c 2}$ becomes slower as $T$ is decreased. (iii) $H_{c 2}$ increases very rapidly as the pressure approaches the critical value, though, by contrast, the pressure dependence of $T_{c}$ is moderate.

The absence of the Pauli depairing effect in these systems is consistent with the previous theoretical predictions ${ }^{14}$. However, the origins of the strong suppres- sion of the orbital depairing effect and the other extraordinary behaviors (ii) and (iii) have not been elucidated so far. It is particularly important to clarify how these unusual properties are related to quantum criticality and also what kind of role is played by the noncentrosymmetry in the compounds $\mathrm{CeRh}(\mathrm{Ir}) \mathrm{Si}_{3}$. The answers to the questions should certainly provide us with deep understanding of superconductivity for strongly correlated electrons near quantum criticality.

In this letter, we demonstrate that the above striking features are naturally understood in terms of the strongly enhanced pairing interaction due to spin fluctuations in the vicinity of the magnetic QCP. Our results reveal that the above features experimentally observed for $\mathrm{CeRhSi}_{3}$ and $\mathrm{CeIrSi}_{3}$ are not specific to these noncentrosymmetric superconductors, but rather intrinsic and universal in strong coupling superconductivity caused by critical fluctuations, as long as the Pauli depairing effect is suppressed by some other mechanisms such as the lack of inversion center of the crystal structure as in the case of $\mathrm{CeRh}(\mathrm{Ir}) \mathrm{Si}_{3}$. Therefore, the unusual properties found for the upper critical fields uniquely characterize the interplay between quantum criticality and spin-fluctuationmediated Cooper pairings.

We focus on the low energy quasiparticles in the clean limit mainly formed by $f$-electrons and approximate the system by the following effective single-band tightbinding model,

$$
\begin{aligned}
S & =S_{0}+S_{\mathrm{SF}}, \\
S_{0} & =\sum_{k} c_{k}^{\dagger}\left(-i \omega_{n}+\varepsilon(\boldsymbol{k})\right) c_{k}+\sum_{k} c_{k}^{\dagger} \alpha \mathcal{L}_{0}^{H}(\boldsymbol{k}) \cdot \boldsymbol{\sigma} c_{k}, \\
S_{\mathrm{SF}} & =-\sum_{k k^{\prime} q} \frac{g^{2}}{6} \chi(q) \boldsymbol{\sigma}_{\alpha \alpha^{\prime}} \cdot \boldsymbol{\sigma}_{\beta \beta^{\prime}} c_{k+q \alpha}^{\dagger} c_{k \alpha^{\prime}} c_{k^{\prime}-q \beta}^{\dagger} c_{k^{\prime} \beta^{\prime}},
\end{aligned}
$$

where $c_{k}=\left(c_{k \uparrow}, c_{k \downarrow}\right)^{t}$ is the annihilation operator of the Kramers doublet. Here we have introduced the notation $k=\left(i \omega_{n}, \boldsymbol{k}\right)$. The lack of inversion symmetry leads to the spin-orbit interaction which is rep- 
resented in the second term with the Zeeman splitting. The third term is the electron-electron interactions through spin fluctuations. For $\mathrm{CeRhSi}_{3}$ and $\mathrm{CeIrSi}_{3}$ which have body centered tetragonal lattice structures, the dispersion relation and the spin-orbit interaction are given by $\varepsilon(\boldsymbol{k})=-2 t_{1}\left(\cos k_{x}+\cos k_{y}\right)+$ $4 t_{2} \cos k_{x} \cos k_{y}-8 t_{3} \cos \left(k_{x} / 2\right) \cos \left(k_{y} / 2\right) \cos k_{z}-\mu$ and $\alpha \mathcal{L}_{0}^{H}=\left(\alpha \sin k_{y},-\alpha \sin k_{x},-\mu_{B} H\right)$. We fix the parameters as $\left(t_{1}, t_{2}, t_{3}, n, \alpha\right)=(1.0,0.475,0.3,1.05,0.5)$ by taking $t_{1}$ as the energy unit. The Fermi surface determined by these parameters is in qualitative agreement with the band calculation and can reproduce the peak structures of the momentum-dependent susceptibility observed by the neutron scattering experiments 16,17,18. To describe the strong spin fluctuations near the QCP, we phenomenologically introduce the effective interaction between quasiparticles $3,19,20,21$,

$$
\chi\left(i \nu_{n}, \boldsymbol{q}\right)=\sum_{a} \frac{\chi_{0} \xi^{2}}{1+\xi^{2}\left(\boldsymbol{q}-\boldsymbol{Q}_{a}\right)^{2}+\left|\nu_{n}\right| /\left(\Gamma_{0} \xi^{-2}\right)}
$$

and $\xi(T)=\frac{\tilde{\xi}}{\sqrt{T+\theta}}$, where $\chi_{0}$ and $\Gamma_{0}$ are respectively the susceptibility and the energy scale of spin fluctuations without strong correlations. These quantities are renormalized through the coherence length $\xi(T)$ as the system approaches the QCP. The critical exponent of $\xi$ is the mean field value $1 / 2$ and $\theta$ is considered to decrease monotonically as the applied pressure approaches the critical value for the AF order ${ }^{19,20}$. The temperature dependence of $\xi$ is also consistent with the recent NMR experiment for $\mathrm{CeIrSi}_{3} 22$. The propagating vectors are $\boldsymbol{Q}_{1}=( \pm 0.43 \pi, 0,0.5 \pi), \boldsymbol{Q}_{2}=(0, \pm 0.43 \pi, 0.5 \pi)$ according to the neutron scattering experiments 17 . In this study, we fix the parameters in $\chi\left(i \nu_{n}, \boldsymbol{q}\right)$ as $\Gamma_{0}=3.6$ and $\tilde{\xi}=0.4647$. The former is of the same order as the Fermi energy and the latter is determined so that the maximum of $\xi$ would be $\xi_{\max } \sim \delta k^{-1}$, where $\delta k$ is the interstice of the $\boldsymbol{k}$-mesh of the Brillouin zone used in our numerical calculations.

To describe the quasiparticles in the strong coupling regime, we introduce the normal selfenergy up to the first order in $g^{2} \chi_{0}$ which is given by $\Sigma_{s_{1} s_{2}}(k)=$ $\frac{T}{N} \sum_{k^{\prime}} g^{2} \chi\left(k-k^{\prime}\right) G_{s_{1} s_{2}}^{0}\left(k^{\prime}\right) \delta_{s_{1} s_{2}}$, neglecting off diagonal elements which are not important in the present study. $G^{0}$ is the Green's function for noninteracting quasiparticles with spin indices $s_{1}, s_{2}$. Re $\Sigma$ only gives the deformation of the Fermi surface while $\operatorname{Im} \Sigma$ gives two important effects to the quasiparticles around the Fermi level; the mass enhancement by the factor $z^{-1}=1-(\operatorname{Im} \Sigma(\pi T)-$ $\operatorname{Im} \Sigma(-\pi T)) / 2 \pi T$ and the damping factor $\gamma=-\operatorname{Im} \Sigma$. It is reasonable to consider that $\varepsilon(\boldsymbol{k})$ already includes the shift due to $\operatorname{Re} \Sigma$ and to replace $\varepsilon(\boldsymbol{k})+\operatorname{Re} \Sigma(k) \rightarrow \varepsilon(\boldsymbol{k})$. We also neglect the change in $\mathcal{L}_{0}^{H}$ by the normal selfenergy, which has little influence in the discussion of the upper critical fields. We note that the coupling constant $g$ should be regarded as an effective one renormalized by the vertex corrections ${ }^{23,24}$.

To study the transition temperatures under applied fields, we use the linearlized Eliashberg equations where the Green's functions of the quasiparticles depend on the vector potential $\boldsymbol{A}$. We employ the familiar quasiclassical approximation for the Green's function $G\left(i \omega_{n}, \boldsymbol{x}, \boldsymbol{y} ; \boldsymbol{A}\right)=$ $e^{i e \varphi(\boldsymbol{x}, \boldsymbol{y})} G\left(i \omega_{n}, \boldsymbol{x}-\boldsymbol{y} ; \boldsymbol{A}=0\right), \varphi(\boldsymbol{x}, \boldsymbol{y})=\int_{\boldsymbol{y}}^{\boldsymbol{x}} \boldsymbol{A}(\boldsymbol{s}) d \boldsymbol{s}$ which is legitimate for $k_{F} l_{H} \gg 1$ where $k_{F}$ is the Fermi wave number and $l_{H}$ the magnetic length. We take only the $N=0$ Landau level because, as shown below, $H_{c 2}$ is mainly determined by the orbital depairing effect. The gap function is approximated by $\Delta_{\alpha \alpha^{\prime}}(k ; \boldsymbol{R})=$ $\Delta_{\alpha \alpha^{\prime}}(k) \phi_{0}\left(R_{x}, R_{y}\right)$, where $\phi_{0}$ is the lowest Landau level wave function. The resulting Eliashberg equations for $\Delta(k)$ with applied field $\boldsymbol{H}=(0,0, H)$ are,

$$
\begin{aligned}
& \Delta_{\alpha \alpha^{\prime}}(k)=\frac{T}{N} \sum_{k^{\prime}, \beta \beta^{\prime} \gamma \gamma^{\prime}} V_{\alpha \alpha^{\prime}, \beta \beta^{\prime}}\left(k, k^{\prime}\right) \\
& \sum_{\tau= \pm}\left(\frac{1+\tau \hat{\mathcal{L}}_{0}^{H}(\boldsymbol{k}) \cdot \boldsymbol{\sigma}}{2}\right)_{\beta \gamma} i \operatorname{sgn}\left(\tilde{\omega}^{\prime}\right) \\
& \left(\frac{2}{a_{\tau}\left(\boldsymbol{k}^{\prime}\right)}\right)^{1 / 2} f\left(\frac{b_{\tau}\left(k^{\prime}\right)}{\sqrt{2 a_{\tau}\left(\boldsymbol{k}^{\prime}\right)}}\right) G_{\beta^{\prime} \gamma^{\prime}}\left(-k^{\prime}\right) \Delta_{\gamma \gamma^{\prime}}\left(k^{\prime}\right)(1)
\end{aligned}
$$

where $\hat{\mathcal{L}}_{0}^{H}(\boldsymbol{k})=\mathcal{L}_{0}^{H}(\boldsymbol{k}) / \sqrt{\sum_{i=1}^{3} \mathcal{L}_{0 i}^{H}(\boldsymbol{k})^{2}}, \tilde{\omega}(k)=\omega_{n}-$ $\operatorname{Im} \Sigma(k), a_{\tau}(\boldsymbol{k})=\sqrt{|e| H}\left(v_{\tau x}(\boldsymbol{k})^{2}+v_{\tau y}(\boldsymbol{k})^{2}\right), b_{\tau}(k)=$ $|\omega \tilde{\omega}(k)|+i \operatorname{sgn}(\tilde{\omega}) \varepsilon_{\tau}(\boldsymbol{k})$, with the dispersion $\varepsilon_{\tau}(\boldsymbol{k})=\varepsilon(\boldsymbol{k})+$ $\tau \alpha \sqrt{\sum_{i=1}^{3} \mathcal{L}_{0 i}^{H}(\boldsymbol{k})^{2}}$ and the velocity $\boldsymbol{v}_{\tau}(\boldsymbol{k})=\nabla \varepsilon_{\tau}(\boldsymbol{k})$. $f(z)$ is defined as $f(z)=\frac{\sqrt{\pi}}{2} e^{z^{2}} \operatorname{erfc}(z)$. Here, we have neglected the $\Pi$ operators in $\gamma=-\operatorname{Im} \Sigma$ because $\nabla \gamma \cdot \Pi$ represents the contribution to $H_{c 2}$ from the anisotropy of the lifetime of the quasiparticles on the Fermi surface and is of secondary importance compared to $\gamma$ itself. The velocity can be renormalized by the factor $z(\boldsymbol{k})$, leading to enhancement of $H_{c 2}$. The effective pairing interaction $V$ is evaluated at the lowest order in $g^{2} \chi_{0}$,

$$
\begin{aligned}
& V_{s s, s s}\left(k, k^{\prime}\right)=-\frac{1}{6} g^{2} \chi\left(k-k^{\prime}\right)+\frac{1}{6} g^{2} \chi\left(k+k^{\prime}\right), \\
& V_{s \bar{s}, s \bar{s}}\left(k, k^{\prime}\right)=\frac{1}{6} g^{2} \chi\left(k-k^{\prime}\right)+\frac{1}{3} g^{2} \chi\left(k+k^{\prime}\right),
\end{aligned}
$$

$V_{s \bar{s}, \bar{s} s}\left(k, k^{\prime}\right)=-V_{s \bar{s}, s \bar{s}}\left(k, k^{\prime}\right)$ and the other components are zero. The applied fields will not affect $V$, because $\Gamma_{0}=3.6$ is large enough compared to the energy scale of the applied fields.

By solving eq.(1) at $H=0$, we find that the $A_{1}$ symmetric superconducting state is most stable among the five irreducible representations of point group $\mathrm{C}_{4 v}$, which is consistent with the previous study $\stackrel{16}{ }$. In the following, we focus on this state. The $\mathrm{A}_{1}$ symmetric order parameter is in the form of $\Delta\left(i \omega_{n}, \boldsymbol{k}\right)=\left[\Delta_{s}\left(i \omega_{n}\right) d_{0}(\boldsymbol{k})+\right.$ $\left.\Delta_{t}\left(i \omega_{n}\right) \boldsymbol{d}(\boldsymbol{k}) \cdot \boldsymbol{\sigma}\right] i \sigma_{2}$ with $d_{0}=\cos \left(2 k_{z}\right), \boldsymbol{d}=\cos \left(2 k_{z}\right) \mathcal{L}_{0}$. In our model, however, the amplitude of the triplet part $\Delta_{t}$ is so small $\left(\lesssim 0.01 \Delta_{s}\right)$ that we will neglect it in the following. In Fig 1, we show the transition temperatures $T_{c}$ for the $\mathrm{A}_{1}$ symmetric superconducting state at $H=0$ 


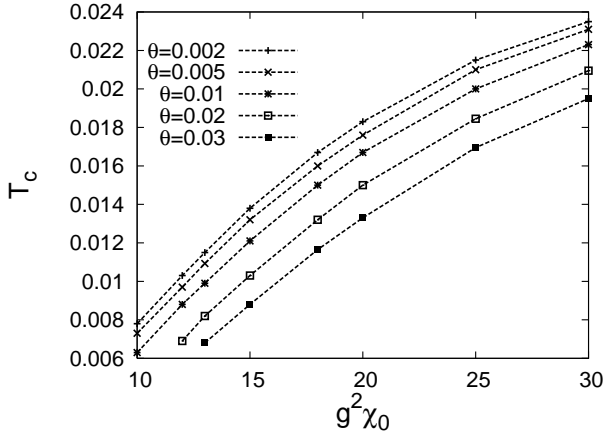

FIG. 1: Transition temperatures for the extended $s$-wave state as functions of $g^{2} \chi_{0}$ for several $\theta$.

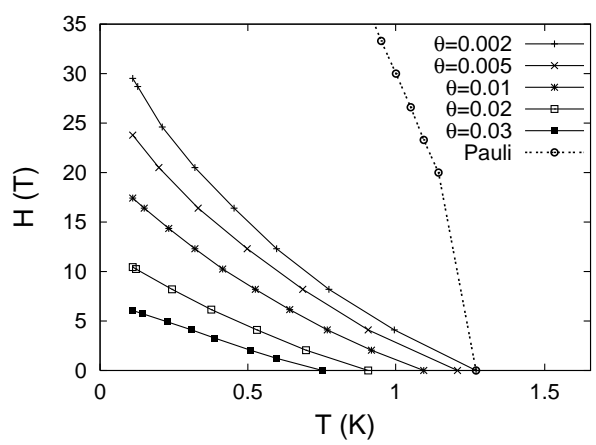

FIG. 2: Temperature dependence of the upper critical fields for the extended $s$-wave state. The dotted line with open circles represents the Pauli limiting field for $\theta=0.002$ and the others the orbital limiting fields for several $\theta$. The unit of $\theta$ is $t_{1}=113 \mathrm{~K}$.

as functions of $g^{2} \chi_{0}$ for several $\theta$. The transition temperatures saturate for large $g^{2} \chi_{0}$ because of the strong coupling effects. Their dependence on $\theta$ is quite weak.

We now turn to the case with $H \neq 0$. In Fig 2, we show upper critical fields as functions of temperature for several $\theta$ at $g^{2} \chi_{0}=13$. For this value of $g^{2} \chi_{0}$, the renormalization factor $\left.z^{-1}\right|_{T=0.01}$ is $\sim 2$, which indicates that the system is in the strong coupling regime. In Fig.2, we use the following values of the parameters; (lattice constant $)=4 \AA^{9,11}$ and $t_{1}=113 \mathrm{~K}$. The latter is obtained by identifying the calculated maximum transition temperature $T_{c}=0.0115$ as $1.3 \mathrm{~K}$ which is the averaged value

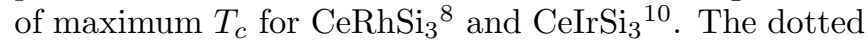
line with open circles in Fig.2 corresponds to the Pauli limiting field for $\theta / t_{1}=0.002$ and the other lines to the orbital limiting fields for several $\theta$. In the present parameters, transition temperatures are typically $T_{c} \simeq 0.01 t_{1}$ and thus the spin-orbit coupling constant is very large compared to $T_{c}\left(\alpha=0.5 t_{c} \simeq 50 T_{c}\right)$, leading to $H_{c 2}^{\text {Pauli }}$ largely exceeding $H_{c 2}^{\text {orb }}$. Note that, with sufficiently large $\alpha, H_{c 2}^{\text {Pauli }}(0)$ calculated from the Eliashberg equations coincides with the one obtained from thermodynamic considerations $\frac{15}{\underline{ }}$. From the result, we can conclude that,

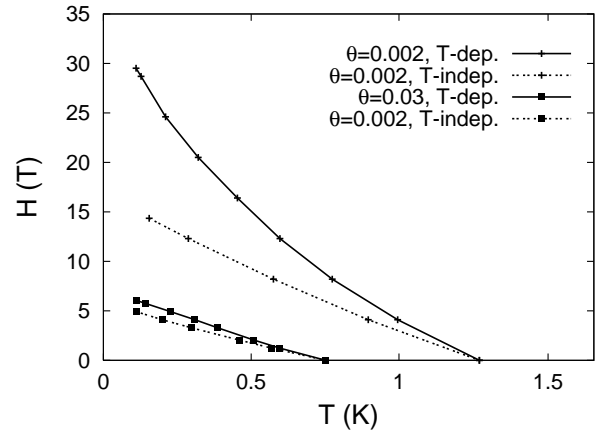

FIG. 3: $H_{c 2}^{\text {orb }}$ versus $T$ calculated with $T$-dependent $\xi$ (solid) and $T$-independent $\xi$ (dotted) for $\theta=0.002,0.03$.

for sufficiently large $\alpha \gg T_{c}$, the upper critical fields are determined by the orbital limiting fields in accordance with the previous studies ${ }^{14.15}$. A remarkable point in Fig 2 is that the orbital limiting fields are enhanced as $\theta$ is decreased, and reach $30 \mathrm{~T}$ for sufficiently small $\theta$ even when $T_{c}(H=0) \sim 1 \mathrm{~K}$, which well explains the experimentally observed huge $H_{c 2}$ for $\mathrm{CeRhSi}_{3}$ and $\mathrm{CeIrSi}_{3}$. Also, the $H_{c 2}$ curve has a upward curvature, which is consistent with the experimental observations. These results verify that the superconductivity in these systems is mediated by the strong antiferromagnetic spin fluctuations near the QCP.

The origin of the colossal enhancement in $H_{c 2}^{\text {orb }}$ is understood as follows. As the temperature approaches absolute zero, the antiferromagnetic correlation length $\xi(T)$ increases, leading to the enhancement of the effective pairing interaction $V \propto \xi(T)^{2}$, while the pair breaking effect due to the quasiparticle damping $\operatorname{Im} \Sigma(T)$ is suppressed. As a result, the coherence length of the superconducting state is extremely reduced at low temperatures. This behavior is significant especially for small $\theta$, giving rise to the huge values of $H_{c 2}^{\text {orb }}$, as seen in Fig, 2 , To demonstrate the importance of the rapid increase in $\xi(T)$, we also calculate $H_{c 2}$ by assuming that $\xi(T)$ is independent of temperature and has a constant value, $\xi=\xi\left(T_{c}\right)$ (Fig. 3). We see that the $H_{c 2}^{\text {orb }}$ curves calculated with temperature independent $\xi$ exhibit neither significant upturn nor enhanced values of $H_{c 2}(T)$ at low temperatures. The comparison of these results with those computed with the correct $\xi(T)$, as shown in Fig. 3, concludes that the rapid increase in $\xi(T)$ is essential to obtain the huge values of $H_{c 2}^{\text {orb }}$ as well as the characteristic upturn profile at low temperatures.

Another remarkable feature of the experimental observations for $\mathrm{CeRhSi}_{3}$ and $\mathrm{CeIrSi}_{3}$ is the strong pressure dependence of $H_{c 2} \frac{12,13}{2}$; i.e. $H_{c 2}$ increases explosively as the pressure approaches the critical value, while the pressure dependence of $T_{c}$ is moderate. To discuss this point in detail, we focus on $T_{c}(H=0)$ and $H_{c 2}$ at low temperature as functions of $\theta$. Figure 4 shows the $\theta$ dependence of the transition temperatures at $H=0$ 


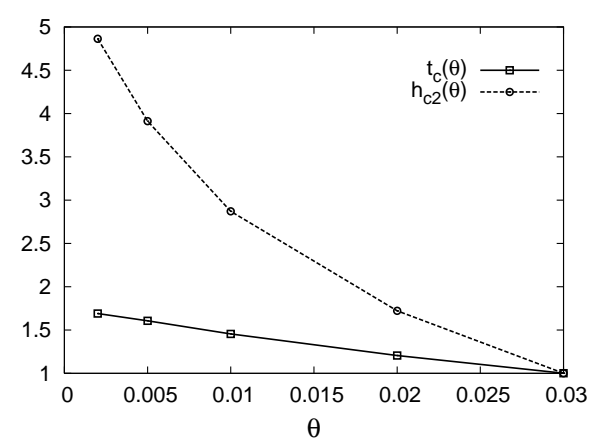

FIG. 4: $\theta$ dependence of the transition temperature $t_{c}(\theta)$ and the upper critical field $h_{c 2}(\theta)$. The definitions of $t_{c}(\theta)$ and $h_{c 2}(\theta)$ are given in the text.

and the upper critical fields at $T=T_{\min } \equiv 0.001$ normalized by the values calculated with $\theta=\theta_{\max } \equiv 0.03$; $t_{c}(\theta) \equiv T_{c}(H=0, \theta) / T_{c}\left(H=0, \theta=\theta_{\max }\right)$ and $h_{c 2}(\theta) \equiv$ $H_{c 2}\left(T=T_{\min }, \theta\right) / H_{c 2}\left(T=T_{\min }, \theta=\theta_{\max }\right)$. We see that $t_{c}(\theta)$ is slightly changed as $\theta$ is decreased. By contrast, $h_{c 2}(\theta)$ is strongly enhanced, which is consistent with the above-mentioned experimental results ${ }^{12,13}$. These behaviors are understood as a result of the strongly enhanced pairing interaction at low temperatures in the vicinity of the QCP. Although the increase of $T_{c}(H=0)$ near the QCP (i.e. $\theta=0$ ) is considerably suppressed by the pair breaking effects due to the inelastic scatterings with spin fluctuations, $H_{c 2}$ at low temperatures is not seriously affected by them, resulting in the strong enhancement of $H_{c 2}$ for $\theta \sim 0$.

We have theoretically clarified the origins of several puzzling phenomena posed experimentally for $\mathrm{CeRh}(\mathrm{Ir}) \mathrm{Si}_{3}$. Here we wish to stress that the extraordinary properties of $H_{c 2}$ discussed here are not specific to these compounds, but are inherent in the orbital limited superconductors caused by spin fluctuations in the vicinity of the QCP. However, such anomalous behaviors of $H_{c 2}$ have not been observed so far in any other systems than $\mathrm{CeRh}(\mathrm{Ir}) \mathrm{Si}_{3}$, although there are many superconducting systems which are believed to be located in the vicinity of a magnetic QCP. The key to resolve this apparent contradiction is the lack of inversion symmetry. In the presence of the sufficiently large anisotropic spin-orbit interaction, as discussed above, the dominant mechanism which determines the properties of $H_{c 2}$ is the orbital depairing. In addition, generally, the orbital limiting fields are much more influenced by the electron correlations than the Pauli ones. In ordinary centrosymmetric superconductors, the enhanced strong coupling effects on $H_{c 2}$ near a QCP, which could exist in principle, are masked by the Pauli depairing effects. The noncentrosymmetric heavy fermion superconductors $\mathrm{CeRhSi}_{3}$ and $\mathrm{CeIrSi}_{3}$ with large anisotropic spin-orbit interactions are the first systems in which $H_{c 2}$ directly exhibits the enhanced strong coupling effects due to spin fluctuations near the QCP. We thus naturally expect that the anomalous properties of $H_{c 2}$ could be more generally observed in other strong-coupling superconductors caused by critical spin fluctuations if the Pauli depairing effect could be suppressed by some other mechanisms.

In summary, we have studied the strong coupling effects on the upper critical fields in $\mathrm{CeRhSi}_{3}$ and $\mathrm{CeIrSi}_{3}$. We have found that the upper critical fields are extremely enhanced by critical spin fluctuations near the QCP, which successfully explains the recent experimental observations. We have elucidated that the huge values of $H_{c 2}$ and its strong dependence on the applied pressure is a universal property inherent in the orbital limited superconductors near the $\mathrm{QCP}$.

We thank N. Kimura, R. Settai and Y.Ōnuki for valuable discussions. Numerical calculations were partially performed at the Yukawa institute. This work is partly supported by the Grant-in-Aids for Scientific Research from MEXT of Japan (Grant No.18540347, Grant No.19014009, Grant No.19014013, Grant No.19052003, and Grant No.20029013). Y. Tada is supported by JSPS Research Fellowships for Young Scientists.
* Electronic address: tada@scphys.kyoto-u.ac.jp

1 For a review, see, e.g. Y. Kitaoka, H. Mukuda, M. Yashima, and A. Harada, J. Phys. Soc. Jpn. 76, 051001 (2007); J. L. Sarrao and J. D. Thompson, J. Phys. Soc. Jpn. 76, 051013 (2007).

2 K. Miyake, S. Schmitt-Rink and C. M. Varma, Phys. Rev. B 34, 6554 (1986).

3 P. Monthoux and D. Pines, Phys. Rev. Lett. 69, 961 (1992).

4 T. R. Kirkpatrick, D. Belitz, T. Vojta and R. Narayana, Phys. Rev. Lett. 87, 127003 (2001).

5 Z. Wang, W. Mao and K. Bedell, Phys. Rev. Lett. 87, 257001 (2001).

${ }^{6}$ R. Roussev and A. J. Millis, Phys. Rev. B 63, 140504 (2001).
7 A. V. Chubukov, A. M. Finkel'stein, R. Haslinger and D. K. Morr, Phys. Rev. Lett. 90, 077002 (2003).

8 N. Kimura, K. Ito, K. Saitoh, Y. Umeda, H. Aoki, and T. Terashima, Phys. Rev. Lett. 95, 247004 (2005).

9 Y. Muro, M. Ishikawa, K. Hirota, Z. Hiroi, N. Takeda, N. Kimura, and H. Aoki, J. Phys. Soc. Jpn. 76, 033706 (2007).

10 I. Sugitani, Y. Okuda, H. Shishido, T. Yamada, A. Thamizhavel, E. Yamamoto, T. D. Matsuda, Y. Haga, T. Takeuchi, R. Settai, and Y. Ōnuki, J. Phys. Soc. Jpn. 75, 043703 (2006).

11 Y. Okuda, Y. Miyauchi, Y. Ida, Y. Takeda, C. Tonohiro, Y. Oduchi, T. Yamada, N. D. Dung, T. D. Matsuda, Y. Haga, T. Takeuchi, M. Hagiwara, K. Kindo, H. Harima, K. Sugiyama, R. Settai, and Y. Ōnuki, J. Phys. Soc. Jpn. 
76, 044708 (2007).

12 N. Kimura, K. Ito, H. Aoki, S. Uji, and T. Terashima, Phys. Rev. Lett. 98, 197001 (2007).

13 R. Settai, Y. Miyauchi, T. Takeuchi, F. Lévy, I. Siieikin, and Y. Ōnuki, J. Phys. Soc. Jpn. 74, 073705 (2008)

14 P. A. Frigeri, D. F. Agterberg, A. Koga, and M. Sigrist, Phys. Rev. Lett. 92, 097001 (2004).

15 S. Fujimoto, J. Phys. Soc. Jpn. 76, 051008 (2007).

16 Y. Tada, N. Kawakami, and S. Fujimoto, J. Phys. Soc. Jpn. 77, 054707 (2008).

17 N. Aso, H. Miyano, H. Yoshizawa, N. Kimura, T. Komatsubara, and H. Aoki, J. Mag. Mag. Matt. 310, 602 (2007).

18 T. Terashima, Y. Takahide, T. Matsumoto, S. Uji, N.
Kimura, H. Aoki, and H. Harima, Phys. Rev. B 76, 054506 (2007)

19 T. Moriya and K. Ueda, Rep. Prog. Phys. 66, 1299 (2003).

20 A. J. Millis, H. Monien, and D. Pines, Phys. Rev. B 42, 167 (1990).

21 P. Monthoux and G. G. Lonzarich, Phys. Rev. B 59, 14598 (1999).

${ }^{22}$ H. Mukuda, T. Fujii, T. Ohara, A. Harada, M. Yashima, Y. Kitaoka, Y. Okuda, R. Settai, and Y. Ōnuki, Phys. Rev. Lett. 100, 107003 (2008).

23 K. Yonemitsu, J. Phys. Soc. Jpn. 58, 4576 (1989).

24 P. Monthoux, Phys. Rev. B 55, 15261 (1997). 